\title{
Quantum coherence and state conversion: theory and experiment
}

\author{
Kang-Da Wu ${ }^{1,2,5}$, Thomas Theurer ${ }^{3,5}$, Guo-Yong Xiang ${ }^{1,2}$, Chuan-Feng Li ${ }^{1,2}$, Guang-Can Guo ${ }^{1,2}$, Martin B. Plenio ${ }^{3}$ and \\ Alexander Streltsov ${ }^{4 凶}$
}

The resource theory of coherence studies the operational value of superpositions in quantum technologies. A key question in this theory concerns the efficiency of manipulation and interconversion of the resource. Here, we solve this question completely for qubit states by determining the optimal probabilities for mixed-state conversions via stochastic incoherent operations. Extending the discussion to distributed scenarios, we introduce and address the task of assisted incoherent state conversion, where the process is enhanced by making use of correlations with a second party. Building on these results, we demonstrate experimentally that the optimal state-conversion probabilities can be achieved in a linear optics setup. This paves the way towards real world applications of coherence transformations in current quantum technologies.

npj Quantum Information (2020)6:22; https://doi.org/10.1038/s41534-020-0250-z

\section{INTRODUCTION}

Practical constraints on our ability to manipulate physical systems restrict the control we can exert on them. It is, e.g., exceedingly difficult to exchange quantum systems undisturbed over long distances. ${ }^{1}$ When manipulating spatially separated subsystems, effectively, this limits us to Local Operations and Classical Communication (LOCC). Under these operations, it is only possible to prepare certain states, i.e., separable ones. The states that cannot be produced under LOCC are called entangled and are elevated to resources: consuming them allows to implement operations such as quantum-state teleportation ${ }^{2}$ to achieve perfect quantum-state transfer, which would not be possible with LOCC alone. This has important consequences, e.g., in quantum communication and other quantum technologies, but also for our understanding of the view of the fundamental laws of nature. ${ }^{1,3-5}$

Entanglement is explored within the framework of quantum resource theories, which can also be used to investigate other non-classical features of quantum mechanics in a systematic way. A concept underlying many facets of non-classicality, including entanglement, is the superposition principle. As a quantum system naturally decoheres in the presence of unavoidable interactions between the system and its environment, ${ }^{6,7}$ superposition is itself a resource, which is studied in the recently developed resource theory of quantum coherence. ${ }^{8-14}$ In this framework, the set of free operations analogous to LOCC in entanglement theory are incoherent operations (IOs), corresponding to quantum measurements which cannot create coherence even if postselection is applied on the individual measurement outcomes. $^{10}$

One of the central questions in any resource theory is the stateconversion problem, i.e., the characterization of the possible interconversion of resources under transformations allowed by the corresponding resource theory. The answer to this question leads to a preorder on the resource states, which determines their usefulness or value in technological applications, as a given state can be used in all protocols that require a state that can be created from it. The state-conversion problem is studied in two opposing limits: in the single-shot regime, where one studies the conversion of a single copy of a state, or in the asymptotic limit, in which one assumes that asymptotically many copies are available. The single-shot state-conversion problem using IOs has been solved for all pure states ${ }^{11,15}$ and for mixed states of a single qubit. ${ }^{12,16,17}$ Asymptotic incoherent conversions were investigated in ref. ${ }^{11}$ In the single-shot regime, a more general problem concerns stochastic state conversions, i.e., finding the optimal probability for probabilistic incoherent conversions between two given quantum states. For conversions between pure states, this question has been addressed in refs. ${ }^{18,19}$ In this work, we provide a full solution to the stochastic incoherent state-conversion problem for qubit systems, the fundamental building blocks in quantum information. This generalizes recent results on singleshot coherence theory ${ }^{12,16-22}$ and single-shot resource theories in general, ${ }^{23,24}$ opening new perspectives on how such resources can lead to practical advantages in quantum metrology, ${ }^{25,26}$ quantum algorithms, ${ }^{27,28}$ and even quantum dynamics in biology. ${ }^{29}$

When the technology to build quantum computers becomes available, it is likely that they will appear initially in small numbers. They have complete control over their qubits and can assist a less powerful remote client restricted to IOs. In particular, they can assist him in state conversions, which we study under the name of assisted incoherent state conversion, solving the problem of optimality for two-qubit pure and Werner states. Moreover, we demonstrate an experimental realization of non-unitary IOs using photonic quantum technologies and show their capability of implementing optimal state conversion on qubits, both with and without assistance. This is an important step towards the experimental investigation and systematic manipulation of coherence in quantum technological applications.

${ }^{1}$ CAS Key Laboratory of Quantum Information, University of Science and Technology of China, Hefei 230026, China. ${ }^{2}$ CAS Center For Excellence in Quantum Information and Quantum Physics, University of Science and Technology of China, Hefei 230026, China. ${ }^{3}$ Institute of Theoretical Physics, Universität Ulm, Albert-Einstein-Allee 11, D-89069 Ulm, Germany. ${ }^{4}$ Centre for Quantum Optical Technologies, Centre of New Technologies, University of Warsaw, Banacha 2c, 02-097, Warsaw, Poland. ${ }^{5}$ These authors contributed equally: Kang-Da Wu, Thomas Theurer. ${ }^{凶}$ email: gyxiang@ustc.edu.cn; a.streltsov@cent.uw.edu.pl 


\section{RESULTS}

Theoretical framework

In this section, we describe the foundations of this work. In the resource theory of coherence, an orthonormal basis of states $\{|i\rangle\}$, usually motivated on physical grounds as being easy to synthesize or store, are considered classical. Any mixture of such states,

$\rho=\sum_{i} p_{i}|i\rangle\langle i|$,

is referred to as "free" and is termed incoherent, similar to probability distributions on classical states. The free operations are referred to as IOs: ${ }^{10}$ these are quantum transformations $\Lambda$, which admit an incoherent Kraus decomposition

$\Lambda[\rho]=\sum_{i} K_{i} \rho K_{i}^{\dagger}$

with incoherent Kraus operators $K_{i}$, i.e., $K_{i}|m\rangle \sim|n\rangle$ for incoherent states $|m\rangle$ and $|n\rangle$. IOs admit a natural interpretation as quantum measurements which cannot create coherence even if postselection is applied to the individual measurement outcomes $i$ identified with the Kraus operators $K_{i}$. Most of the analysis presented in the following can be reduced to the mathematically simpler family of strictly incoherent operations (SIOs). These are operations that can be decomposed into strictly incoherent Kraus operators $K_{i}$, which are defined by the property that both $K_{i}$ and $K_{i}^{\dagger}$ are incoherent, and correspond to quantum processes that do not use coherence. ${ }^{11,13}$

A general deterministic operation has the form (2), where the Kraus operators $K_{i}$ fulfill the completeness condition $\sum_{i} K_{i}^{\dagger} K_{i}=1$. To implement a stochastic IO, we formally postselect a deterministic $I O$ according to the measurement outcomes $i$. Now, assume we deal with a stochastic operation that can be decomposed into incoherent Kraus operators that are not necessarily complete, i.e., $\sum_{i} K_{i}^{\dagger} K_{i} \leq \mathbb{1}$. If we want to call this operation incoherent, we must ensure that it can be seen as part of a deterministic 10 , otherwise we would simply disregard the nonfree part of a costly operation. The fact that this is always possible has been shown in ref. ${ }^{30}$ Therefore, we call all stochastic operations that can be decomposed into incoherent Kraus operators incoherent as well. As the following Proposition tells, the same holds true for SIOs.

Proposition 1. Every stochastic quantum operation that can be decomposed into strictly incoherent Kraus operators is part of a deterministic SIO.

The proof can be found in the Supplementary Material.

In this work, we will solve the problem of state conversion for qubits under the restricted sets of operations $I O$ and SIO theoretically. To do this, we make use of the following Theorem proven in the Supplementary Material, stating that the two problems are equivalent.

Theorem 2. Let $\rho$ and $\sigma$ be states of a single qubit. The following statements are equivalent:

(1) There exists an IO transforming $\rho$ with probability $p$ to $\sigma$.

(2) There exists a SIO transforming $\rho$ with probability $p$ to $\sigma$.

Then, we extend our analysis to the problem of assisted incoherent state conversion, which we introduce now as a game between two parties, Alice and Bob. Initially, they share a bipartite quantum state $\rho^{A B}$ and the aim of the game is to establish a certain state $\sigma^{B}$ on Bob's side. Clearly, if all quantum transformations were allowed locally, Bob could achieve this task by simply erasing his local system and preparing the desired state $\sigma^{B}$. However, the situation changes if Bob is constrained to local IOs: in this case, he cannot prepare the state $\sigma^{B}$ if the state has coherence. Moreover, as we will show later, correlations in the joint state $\rho^{A B}$ can be used to enhance Bob's conversion possibilities, if Alice assists Bob by measuring her particle and communicating the measurement outcome.

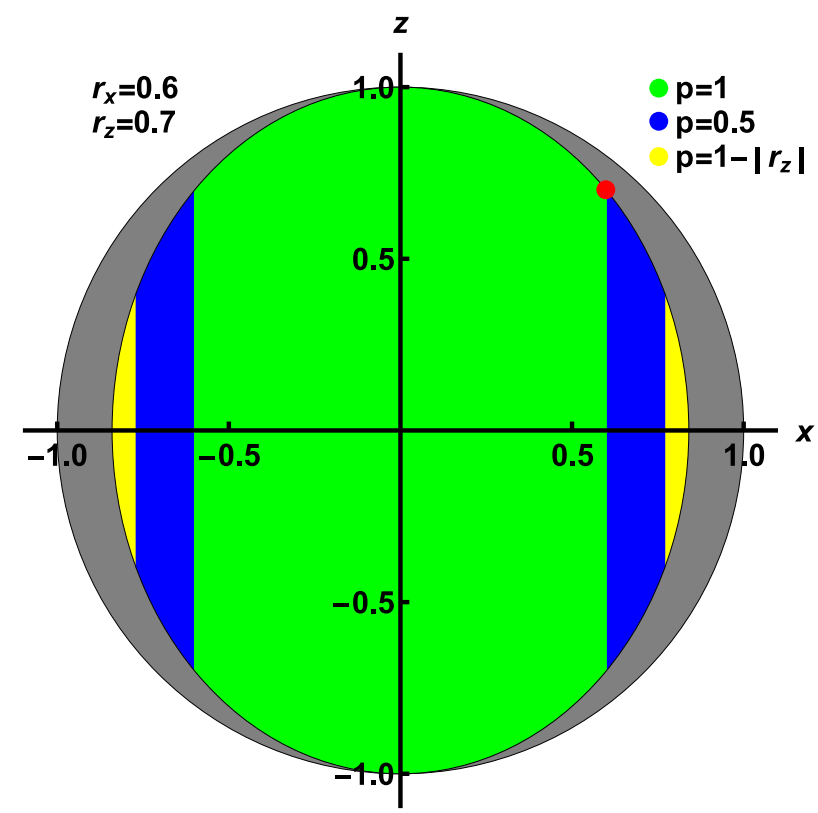

Fig. 1 Incoherent state conversion. Illustration of Theorem 3 in the $x-z$ plane of the Bloch sphere. For an initial state with $r_{x}=r=0.6$ and $r_{z}=0.7$, which is depicted by the red dot, the reachable regions for three different probabilities $p$ are shown. The regions that are reachable with lower probability include the ones reachable with higher probability. The gray region cannot be reached with non-zero probability.

As most of this work is concerned with qubits, we will make frequent use of the Bloch representation, stating that every qubit state $\rho$ can be represented by a subnormalized vector $\boldsymbol{r}=$ $\left(r_{x}, r_{y}, r_{z}\right)$ through $\rho=(\mathbb{1}+\boldsymbol{r} \cdot \boldsymbol{\sigma}) / 2$, where $\boldsymbol{\sigma}$ represents a vector containing the Pauli matrices. As done above, we denote density operators by small Greek letters and their Bloch vectors by the respective small Latin letter. Throughout the paper, we assume the eigenbasis of $\sigma_{z}$ to be incoherent. Then, rotations about the $z$-axis of the Bloch sphere and their inverse are both in SIO and in $I O$, leading to an invariance of conversion probabilities under these rotations. This makes it very convenient to introduce the quantity

$r=\sqrt{r_{x}^{2}+r_{y}^{2}}$

corresponding to the distance of the state to the incoherent axis.

\section{Optimal conversion without assistance}

For a general qubit state $\rho$, the exact shape of the state space that can be achieved by IOs is described in the following Theorem, making use of the Bloch sphere representation introduced above, where $\boldsymbol{r}$ and $\boldsymbol{s}$ are the Bloch vectors of the initial and the final state, respectively.

Theorem 3. A qubit state $\sigma$ is reachable via a stochastic SIO or IO transformation from a fixed initial qubit state $\rho$ with a given probability $p$ if

$r^{2} s_{z}^{2}+\left(1-r_{z}^{2}\right) s^{2} \leq r^{2}$,

$p^{2} s^{2} \leq \frac{r^{2}}{1+\left|r_{z}\right|}\left[2 p-\left(1-\left|r_{z}\right|\right)\right]$

The proof of this Theorem can be found in the Supplementary Material. As shown in Fig. 1, this theorem has a convenient geometrical interpretation on the Bloch sphere: for fixed $\rho$, Eq. (4a) defines an ellipsoid that is independent of $p$ and Eq. (4b) a cylinder that depends on $p$. The states to which $\rho$ can be converted with 
probability $p$ lie inside their intersection. For $p \leq 1-\left|r_{z}\right|$, the ellipsoid is entirely contained in the cylinder and Eq. (4b) is automatically satisfied if Eq. (4a) holds (see proof of Theorem 3). Therefore, lowering the demanded probability of success below $1-\left|r_{z}\right|$ will not increase the set of reachable states. This implies that for mixed $\rho$, there is a discontinuity in the optimal conversion probability $P(\rho \rightarrow \sigma)$ and the states outside the ellipsoid cannot be achieved via stochastic IOs, even with arbitrary little probability.

From Theorem 3, we can deduce the following Corollary:

Corollary 4. The maximal probability $P(\rho \rightarrow \sigma)$ for a successful transformation from a coherent qubit state $\rho$ to a coherent qubit state $\sigma$ using $\mathrm{IO}$ or $\mathrm{SIO}$ is zero if

$r^{2} s_{z}^{2}+\left(1-r_{z}^{2}\right) s^{2}>r^{2}$

and

$\min \left\{\frac{r^{2}}{\left(1+\left|r_{z}\right|\right) s^{2}}\left(1+\sqrt{1-\frac{s^{2}\left(1-r_{z}^{2}\right)}{r^{2}}}\right), 1\right\}$

otherwise.

The proof of this Corollary is given in the Supplementary Material. Moreover, these theoretical results can be extended beyond qubits, leading to necessary conditions for stochastic state conversions.

According to ref. ${ }^{10}$, a coherence measure $C$ is a functional mapping quantum states to the non-negative real numbers that is zero exactly on the incoherent states, convex, and monotonic under selective IOs on average. For every such measure $C$, it holds that $^{18}$

$P(\rho \rightarrow \sigma) \leq \frac{C(\rho)}{C(\sigma)}$.

It is noteworthy that the bounds given in Eq. (7) cannot be used to exclude the existence of a stochastic transformation from $\rho$ to $\sigma$ (unless we have the trivial cases $C(\sigma)=\infty$ or $\rho$ incoherent and $\sigma$ not). However, the first condition in Corollary 4 is a (nontrivial) necessary condition for the existence of a stochastic transformation. In the case of $\mathrm{SIO}$, we can generalize this necessary condition to arbitrary dimensions using the $\Delta$ robustness of coherence $C_{\Delta, R}$ introduced in refs. ${ }^{12,31}$ by

$C_{\Delta, R}(\rho)=\min \left\{t \geq 0 \mid \frac{\rho+t \tau}{1+t} \in \mathcal{I}, \tau \geq 0, \Delta \rho=\Delta \tau\right\}$,

where $\mathcal{I}$ denotes the set of incoherent states.

Theorem 5. A necessary condition for the existence of a stochastic SIO transformation from $\rho$ to $\sigma$ is

$C_{\Delta, R}(\sigma) \leq C_{\Delta, R}(\rho)$.

Again, the proof can be found in the Supplementary Material. As shown in ref. ${ }^{31}$, for the case of qubits, Eq. (9) is equivalent to conditions (4a) and (5), and for higher dimensions, $C_{\Delta, R}$ can be evaluated efficiently using a semi-definite program (see the proof of the Theorem and also ref. ${ }^{32}$ ). The other necessary condition for stochastic transformations on qubits was that the initial state is not incoherent. For higher dimensions, this can be generalized by the statement that the coherence rank or number ${ }^{1,33-35}$ can only decrease under a stochastic IO (and therefore SIO) transformation, which we show now for completeness.

The coherence rank $r_{C}$ of pure states is defined as the number of non-zero coefficients needed to expand the state in the incoherent basis. ${ }^{11,33}$ For mixed states, the coherence rank is defined by ref. ${ }^{34}$

$\left.r_{C}(\rho)=\min \left\{\max _{i} r_{C}\left(\left|\psi_{i}\right\rangle\right)\left|\rho=\sum_{i} p_{i}\right| \psi_{i}\right\rangle\left\langle\psi_{i}\right|, p_{i} \geq 0\right\}$.

It is well known that the coherence rank of a pure state can only decrease under the action of an incoherent Kraus operator. ${ }^{11}$ From this follows the statement directly: let $\left\{p_{i},\left|\psi_{i}\right\rangle\right\}$ be an optimal decomposition of $\rho$ in the sense that $r_{C}(\rho)=\max _{i} r_{C}\left(\left|\psi_{i}\right\rangle\right)$. Applying the Kraus operators of the stochastic IO to the $\left|\psi_{i}\right\rangle$ leads to a decomposition of the final state with the promised property.

Optimal conversion with assistance

We now present our results concerning state conversions with assistance, for pure entangled states and a class of mixed states. The task of assisted incoherent state conversion is equivalent to transforming a shared state $\rho^{A B}$ into a local state $\sigma^{B}$ on Bob's side via local quantum-incoherent operations and classical communication (LQICC). ${ }^{36,37}$ These operations consist of general local operations on Alice's side, local IOs on Bob's side, and the exchange of measurement results via a classical channel. In case only Alice sends information to Bob, we speak of one-way LQICC. The problem of optimal conversion of a general two-qubit entangled state $|\psi\rangle^{A B}$ into an arbitrary local state $\sigma^{B}$ is solved in the following Theorem, which is proven in the Supplementary Material.

Theorem 6. Let Alice and Bob share a pure two-qubit state $|\psi\rangle^{A B}$ and denote Bob's local state by $\rho^{B}$. The maximal probability $P_{\mathrm{a}}\left(|\psi\rangle^{A B} \rightarrow \sigma^{B}\right)$ to prepare the qubit state $\sigma^{B}$ on Bob's side via oneway LQICC is given by

$P_{\mathrm{a}}\left(|\psi\rangle^{A B} \rightarrow \sigma^{B}\right)=\min \left\{1,\left(1-\left|r_{z}\right|\right) \frac{1+\sqrt{1-s^{2}}}{s^{2}}\right\}$,

where $\boldsymbol{r}$ and $\boldsymbol{s}$ are the Bloch vectors of $\rho^{B}$ and $\sigma^{B}$, respectively.

When the state is subjected to noise, the probabilities of assisted incoherent state conversions are reduced. As an example, we consider the two-qubit Werner state,

$\rho_{\mathrm{w}}^{A B}=q_{\mathrm{w}}\left|\phi^{+}\right\rangle\left\langle\phi^{+}\right|+\left(1-q_{\mathrm{w}}\right) \frac{1}{4}$,

with the maximally entangled state $\left|\phi^{+}\right\rangle=(|00\rangle+|11\rangle) / \sqrt{2}$. In this case, the optimal conversion probability is determined by the following Theorem.

Theorem 7. The optimal probability $P_{\mathrm{a}}\left(\rho_{\mathrm{w}}^{A B} \rightarrow \sigma^{B}\right)$ for converting $\rho_{\mathrm{w}}^{A B}$ into the qubit state $\sigma^{B}$ via one-way LQICC is given by,

$P_{\mathrm{a}}\left(\rho_{\mathrm{w}}^{A B} \rightarrow \sigma^{B}\right)=\left\{\begin{array}{cc}1 & \text { if } q_{\mathrm{w}} \geq \frac{s^{2}}{\sqrt{1-s_{z}^{2}}}, \\ 0 & \text { otherwise, }\end{array}\right.$

where $\boldsymbol{s}$ denotes the Bloch vector of $\sigma^{B}$.

This is proven in the Supplementary Material. Determining the optimal assisted incoherent conversion probabilities for general shared mixed states is left open for future work. However, in general, correlations in the joint two-qubit state $\rho^{A B}$ always enhance the conversion possibilities of Bob whenever the state is not quantum incoherent, i.e., not of the form $\rho^{A B}=\sum_{i} p_{i} \rho_{i}^{A} \otimes|i\rangle\left\langle\left. i\right|^{B}\right.$ :

Theorem 8. If Bob's system is a qubit, then for any state $\rho^{A B}$, which is correlated and not quantum incoherent, the set of accessible states for Bob via stochastic one-way LQICC is strictly larger, when compared with $\rho^{A} \otimes \rho^{B}$.

The proof can be found in the Supplementary Material.

Asymptotic state conversion via IO

In the scenario considered so far, we assumed that IOs are applied on one copy of the state $\rho$. In the following, we will extend our investigations to asymptotic conversion scenarios, where IOs are performed on a large number of copies of the state $\rho$. The figure of merit in this setting is the asymptotic conversion rate

$R(\rho \rightarrow \sigma)=\sup \left\{r: \lim _{n \rightarrow \infty}\left(\inf _{\Lambda}\left\|\Lambda\left(\rho^{\otimes n}\right)-\sigma^{\otimes\lfloor n\rfloor\rfloor}\right\|_{1}\right)=0\right\}$,

where $\|M\|_{1}=\operatorname{Tr} \sqrt{M^{\dagger} M}$ is the trace norm, the infimum is performed over all IOs $\Lambda$, and $\lfloor x\rfloor$ is the largest integer smaller 
4

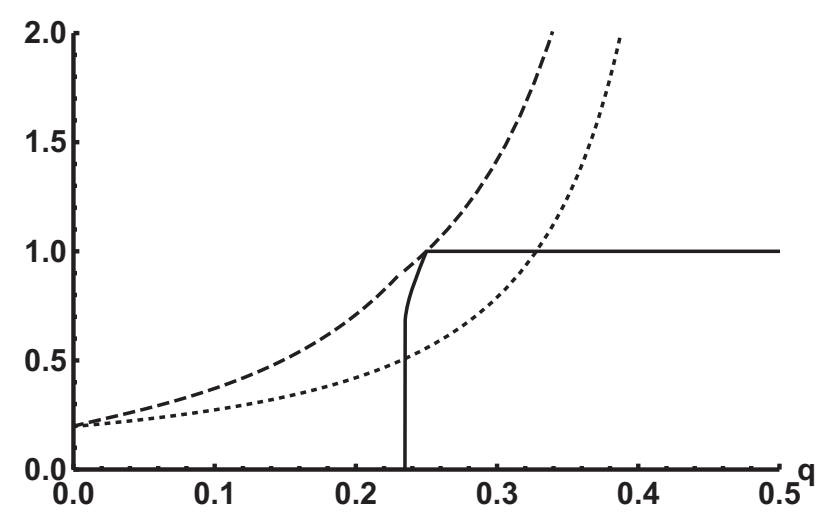

Fig. 2 Comparison of upper and lower bounds on the asymptotic conversion rate. Upper and lower bounds on the asymptotic conversion rate $R(\rho \rightarrow \sigma)$ for states in Eqs. (21) and (22). Dashed line shows the upper bound given by $\min \left\{C_{d}(\rho) / C_{d}(\sigma), C_{c}(\rho) / C_{c}(\sigma)\right\}$, solid line shows the lower bound given by $P(\rho \rightarrow \sigma)$, and dotted line shows the lower bound given by $C_{d}(\rho) / C_{c}(\sigma)$.

or equal to the real number $x$. In words, the quantity $R(\rho \rightarrow \sigma)$ is the maximal rate at which IOs can convert the initial state $\rho$ into the target state $\sigma$, assuming the asymptotic limit of infinitely many initial states on which the $\mathrm{IO}$ can act simultaneously. This quantity should not be confused with the quantity $r$ introduced in Eq. (3) and can exceed 1; e.g., it is possible to convert one coherent state into arbitrary many copies of an incoherent state. In general, the single-shot scenario where one has only access to a single copy of the state under consideration is very different from the asymptotic case of infinitely many copies on which one can act commonly.

It is now important to note that the single copy conversion probability $P(\rho \rightarrow \sigma)$ is a lower bound for the conversion rate, i.e.,

$R(\rho \rightarrow \sigma) \geq P(\rho \rightarrow \sigma)$.

In fact, asymptotic conversion at rate $P(\rho \rightarrow \sigma)$ can be achieved by applying a stochastic 10 on each individual copy of the state $\rho$.

Denoting by $|+\rangle$ the maximally coherent single-qubit state, the distillable coherence ${ }^{11}$ is defined as the maximal rate at which a maximally coherent qubit state can be extracted from $\rho$ using IOs, i.e., $C_{\mathrm{d}}(\rho)=R(\rho \rightarrow|+\rangle\langle+|)$. The complementary quantity, i.e, the minimal rate at which the maximally coherent qubit states need to be consumed to produce the state $\rho$ via IOs, is called the coherence cost ${ }^{11}$ and is formally defined by

$C_{c}(\rho)=\inf \left\{r: \lim _{n \rightarrow \infty}\left(\inf _{\Lambda}\left\|\rho^{\otimes n}-\Lambda\left(|+\rangle\left\langle+\left.\right|^{\otimes\lfloor n\rfloor}\right) \|_{1}\right)=0\right\}\right.\right.$,

where the infimum is again over all IOs. As it was shown in ref. ${ }^{11}$, from these two quantities follow the bounds

$\frac{C_{\mathrm{d}}(\rho)}{C_{\mathrm{c}}(\sigma)} \leq R(\rho \rightarrow \sigma) \leq \min \left\{\frac{C_{\mathrm{d}}(\rho)}{C_{\mathrm{d}}(\sigma)}, \frac{C_{\mathrm{c}}(\rho)}{C_{\mathrm{c}}(\sigma)}\right\}$.

It was also shown in ref. ${ }^{11}$ that the distillable coherence admits the following closed expression:

$C_{\mathrm{d}}(\rho)=S(\Delta[\rho])-S(\rho)$,

where $S(\rho)=-\operatorname{Tr}\left[\rho \log _{2} \rho\right]$ is the von Neumann entropy and $\Delta[\rho]=\sum_{i}|i\rangle\langle i|\rho| i\rangle\langle i|$ is the dephasing operator. Moreover, the coherence cost $C_{c}$ is equal to the coherence of formation $C_{f}{ }^{11}$

$C_{\mathrm{c}}(\rho)=C_{\mathrm{f}}(\rho)=\min \sum_{i} p_{i} S\left(\Delta\left[\psi_{i}\right]\right)$.

Here, the minimization is performed over all pure state decompositions of the state $\rho=\sum_{i} p_{i} \psi_{i}$.

Up until here, the statements concerning asymptotic conversions were valid for general dimensions. From here on, we will specialize them exclusively to qubits. For single-qubit states, Eq.
(19) can be further simplified as follows: ${ }^{38}$

$C_{c}(\rho)=C_{f}(\rho)=h\left(\frac{1+\sqrt{1-4\left|\rho_{01}\right|^{2}}}{2}\right)$,

where $h(x)=-x \log _{2} x-(1-x) \log _{2}(1-x)$ is the binary entropy and $\rho_{01}=\langle 0|\rho| 1\rangle$.

We will now demonstrate the power of these results on a specific example. For this, we consider the following single-qubit state:

$\rho=\left(\begin{array}{cc}\frac{2}{3} & \frac{1}{4} \\ \frac{1}{4} & \frac{1}{3}\end{array}\right)$.

We will study the conversion of $\rho$ into a convex combination of maximally coherent states $| \pm\rangle=(|0\rangle \pm|1\rangle) / \sqrt{2}$, i.e., the final state $\sigma$ has the form

$\sigma=q|+\rangle\langle+|+(1-q)|-\rangle\langle-|$.

In Fig. 2, we compare the aforementioned upper and lower bounds on the state-conversion rate for the states $\rho$ and $\sigma$ in Eqs. (21) and (22). In particular, there exists a range of the parameter $q$ where $P(\rho \rightarrow \sigma)$ (solid line in Fig. 2) is very close to the upper bound $\min \left\{C_{d}(\rho) / C_{d}(\sigma), C_{c}(\rho) / C_{c}(\sigma)\right\}$ (dashed line in Fig. 2). The quality of our bound should also be compared with the lower bound $C_{d}(\rho) / C_{c}(\sigma)$ (dotted line in Fig. 2). The figure clearly shows that the two different lower bounds have their advantages for different values of the parameter $q$ : for $q$ close to $1 / 4$, our new bound is much tighter than the best previously known bound. ${ }^{11}$ If $q$ is below a critical value, the new bound is zero. This corresponds to the region outside the reachable ellipsoid. In addition, the new bound can never exceed 1 and thus the results from ref. ${ }^{11}$ give a better bound when $\sigma$ has a much lower coherence than $\rho$, which corresponds to $q \approx 1 / 2$.

Indeed, we note that for $q=1 / 4$ the conversion probability $P(\rho \rightarrow \sigma)$ coincides with $C_{c}(\rho) / C_{c}(\sigma)$, and in fact both are equal to 1. This implies that the asymptotic conversion rate is given by $R(\rho \rightarrow \sigma)=1$ in this case. We will generalize this observation in the following Theorem.

Theorem 9. Assume qubit states $\rho$ and $\sigma$ obey

$s_{z}^{2} \leq r_{z}^{2} \quad$ and $\quad s=r$.

Then we have $R(\rho \rightarrow \sigma)=1$.

We prove the Theorem in the Supplementary Material, where we also show that it cannot be formulated as an if and only if statement.

We will now apply the methods we developed for studying the irreversibility of coherence theory. For any quantum resource theory, the conversion rate $R$ fulfills the following inequality for any two nonfree states $\rho$ and $\sigma$ :

$R(\rho \rightarrow \sigma) \times R(\sigma \rightarrow \rho) \leq 1$.

The resource theory is called reversible if Eq. (24) is an equality for all nonfree states. Otherwise, the resource theory is called irreversible. Examples for reversible resource theories are the theories of entanglement and coherence, when restricted to pure states only. However, both theories are not reversible for general mixed states. ${ }^{11,39}$ General properties of reversible resource theories have been investigated in refs. ${ }^{40,41}$

In the following, we will study the irreversibility of coherence theory in more detail. In particular, we will investigate which values of distillable coherence $C_{d}$ a single-qubit state can attain, for a fixed amount of coherence cost $C_{c}$. The most interesting family of states in this context is given by $\sigma$ in Eq. (22):

Proposition 10. Among all single-qubit states, the family of states given in Eq. (22) has the minimal distillable coherence $C_{d}$ for a fixed coherence cost $C_{c}$ and vice versa maximal $C_{c}$ for fixed $C_{d}$. 
The Proposition is proven in the Supplementary Material. This result allows us to plot the allowed region of coherence cost and distillable coherence in Fig. 3. The upper curve is given by $C_{d}(\rho)=C_{c}(\rho)$, which is attained if $\rho$ is a pure state. From results in refs., 11,42 it follows directly that the same region is attainable for distillable entanglement and entanglement cost when considering maximally correlated two-qubit states.

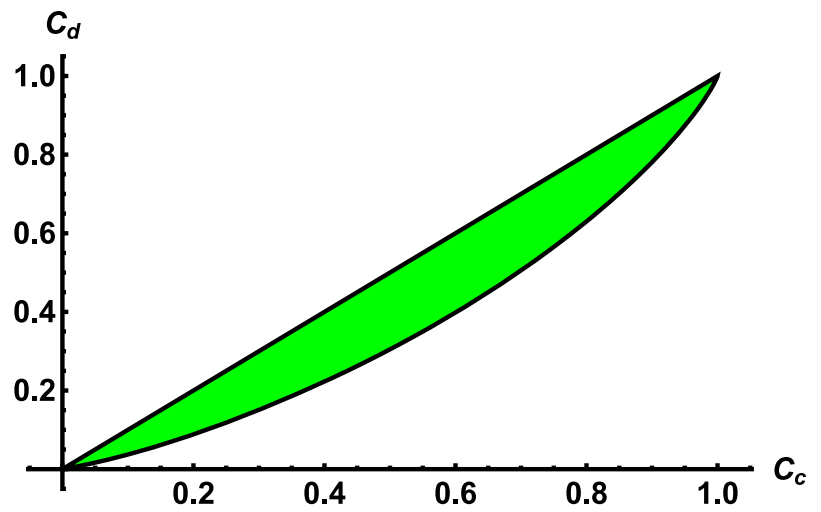

Fig. 3 Irreversibility of coherence theory. Allowed region for distillable coherence $C_{d}$ and coherence cost $C_{c}$ for single-qubit states. The upper curve is given by $C_{d}(\rho)=C_{c}(\rho)$, which is attained for pure states. The lower curve is obtained from the family of states given in Eq. (22), see Proposition 10 and its discussion for details.
Experimental setup and protocols

We experimentally implement the above protocols on several classes of input states, both with and without assistance. The experimental protocols and setup are illustrated in Fig. 4. The setup consists of three modules, which we describe in detail in the following.

In module (I) shown in Fig. $4 \mathrm{~d}$, we can prepare three different classes of states on which we then apply incoherent state conversion with or without assistance. The first class consists of single-qubit states of the form

$\rho^{B}=\frac{1}{2}\left(\mathbb{1}+r_{x} \sigma_{x}+r_{z} \sigma_{z}\right)$

on Bob's side, where $r_{x, z}$ are real numbers and denote $x, z$ Bloch coordinates. The second class consists of pure two-qubit entangled states of the form

$|\Psi\rangle^{A B}=\sqrt{\mu_{0}}|0\rangle^{A}\left|\beta_{0}\right\rangle^{B}+\sqrt{\mu_{1}}|1\rangle^{A}\left|\beta_{1}\right\rangle^{B}$,

where $\mu_{0}, \mu_{1}$ and $\left|\beta_{0}\right\rangle,\left|\beta_{1}\right\rangle$ denote eigenvalues and eigenvectors of Bob's local state. The third class are two-qubit Werner states

$\rho_{w}^{A B}=q_{w}\left|\phi^{+}\right\rangle\left\langle\phi^{+}\right|+\frac{1-q_{w}}{4} \mathbb{1}^{A B}$,

where $\left|\phi^{+}\right\rangle$denotes a maximally entangled state and $q_{\mathrm{w}}$ is the purity of the Werner state.

In particular, two type-I phase-matched $\beta$-barium borate (BBO) crystals, whose optical axes are normal to each other, are pumped by a continuous laser at $404 \mathrm{~nm}$, with a power of $80 \mathrm{~mW}$, for the generation of photon pairs with a central wavelength at $\lambda=$ $808 \mathrm{~nm}$ via a spontaneous parametric down-conversion process. A half-wave plate $(\mathrm{H})$ working at $404 \mathrm{~nm}$ set before the lens and BBO (a)

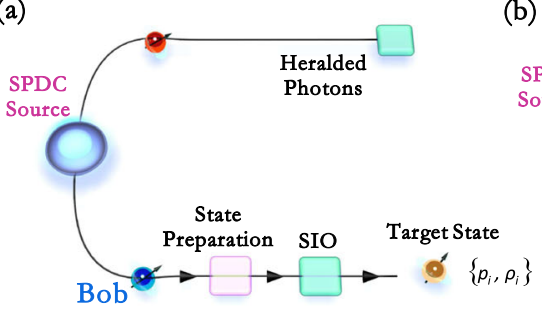

(b)

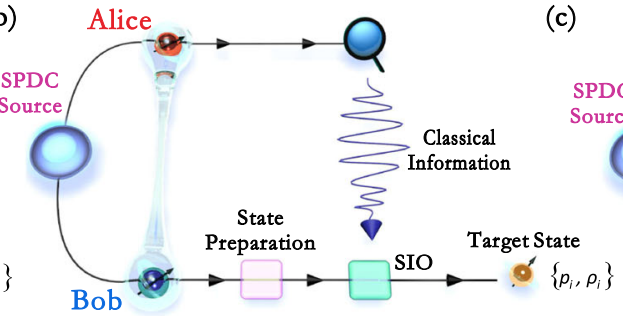

(c)

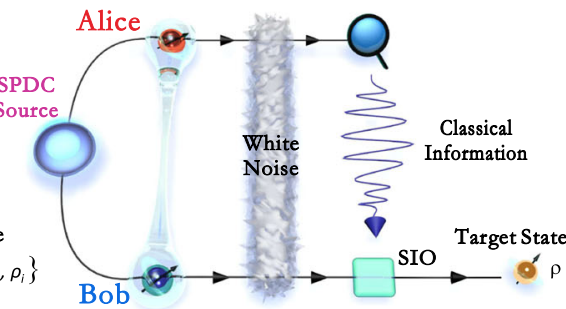

(d)
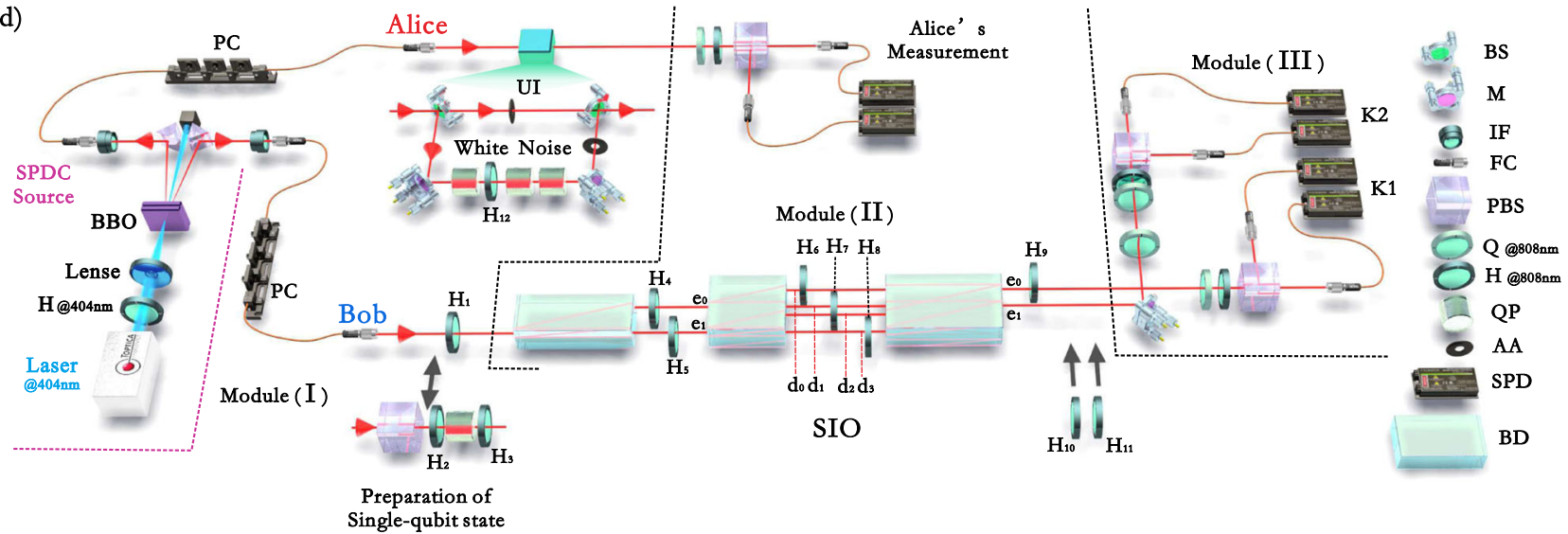

Single-qubit state

Fig. 4 Experimental protocols and setup. In a-c, the three experiments performed in our laboratory are illustrated: a single-qubit conversion without assistance; $\mathbf{b}$ pure entangled two-qubit state conversion with assistance; c noisy two-qubit state conversion with assistance. The whole setup in $\mathbf{d}$ can be divided into three modules: (I) state preparation; (II) incoherent state conversion with or without assistance; and (III) tomography. In (I), we can prepare a class of single-qubit states as in Eq. (25) for Bob, its purification shared with Alice, and a class of Werner states; in (II), we experimentally implement the incoherent operations, both with and without assistance; in (III), we identify the quantum states of Bob. The optical components appearing in the setup are $\beta$-barium borate (BBO), half-wave plate $\left(\mathrm{H}_{i}\right)$, quarter-wave plate $(\mathrm{Q})$, beam displacer (BD), adjustable aperture (AA), interference filter (IF), beam splitter (BS), mirror (M), quartz plate (QP), polarizing beam splitter (PBS), polarization controller (PC), single photon detector (SPD), fiber coupler (FC), and unbalanced interferometer (UI). $K_{122}$ denotes the outcomes of Kraus operators 1(2) implementing the incoherent operation in module (II). 
crystals is used to control the polarization of the pump laser. Two polarization-entangled photons

$$
|\Phi(\theta)\rangle=\cos 2 \theta|H H\rangle+\sin 2 \theta|V V\rangle
$$

are generated and then separately distributed through two singlemode fibers (SMFs), where one represents Bob and the other Alice. Two interference filters with a $3 \mathrm{~nm}$ full width at half maximum are placed to filter out proper transmission peaks. Half-wave plates at both ends of the SMFs are used to control the polarization of both photons. A quarter-wave plate $(\mathrm{Q})$ in Bob's arm is used to compensate the phase for the desired prepared state.

For preparing single-qubit states as in Eq. (25), we set the rotation angle of the $404 \mathrm{~nm} \mathrm{H}$ to $0^{\circ}$, resulting in a state $|H\rangle^{A}|H\rangle^{B}$. By using Alice's photons as trigger, we can experimentally generate a pure incoherent state $|H\rangle^{B}$. We replace $H_{1}$ with a polarizing beam splitter (PBS), a 400 $\lambda$ quartz plate, and another two half-wave plates $\left(\mathrm{H}_{2,3}\right)$ on Bob's side, for generating singlequbit states $\rho^{B}$. The rotation angle of $\mathrm{H}_{2}$ is set to $\gamma_{1}$, rotating the state $|H\rangle$ to another pure state

$\cos 2 \gamma_{1}|H\rangle+\sin 2 \gamma_{1}|V\rangle$.

Then, after the birefringent crystal, the pure state is completely dephased, resulting in an incoherent mixed state

$\cos ^{2} 2 \gamma_{1}|H\rangle\left\langle H\left|+\sin ^{2} 2 \gamma_{1}\right| V\right\rangle\langle V|$.

The rotation angle of $\mathrm{H}_{3}$ is set to $\gamma_{2}$, resulting in the transformation

$$
\begin{aligned}
& |H\rangle \longrightarrow \cos 2 \gamma_{2}|H\rangle+\sin 2 \gamma_{2}|V\rangle, \\
& |V\rangle \longrightarrow \sin 2 \gamma_{2}|H\rangle-\cos 2 \gamma_{2}|V\rangle .
\end{aligned}
$$

The final prepared state reads

$$
\begin{aligned}
\rho^{B}= & \left(\cos ^{2} \gamma_{1} \cos ^{2} \gamma_{2}+\sin ^{2} \gamma_{1} \sin ^{2} \gamma_{2}\right)|H\rangle\langle H| \\
& +\left(\sin ^{2} \gamma_{1} \cos ^{2} \gamma_{2}+\cos ^{2} \gamma_{1} \sin ^{2} \gamma_{2}\right)|V\rangle\langle V| \\
& +\frac{1}{2} \cos 2 \gamma_{1} \sin 2 \gamma_{2}(|H\rangle\langle V|+| V\rangle\langle H|),
\end{aligned}
$$

with Bloch coordinates

$$
\begin{aligned}
& r_{x}=\cos 2 \gamma_{1} \sin 2 \gamma_{2}, \\
& r_{y}=0, \\
& r_{z}=\cos 2 \gamma_{1} \cos 2 \gamma_{2} .
\end{aligned}
$$

Thus, we can prepare the desired single-qubit states as described in Eq. (25).

For generating two-qubit entangled states as given in Eq. (26), we set the rotation angle of the $404 \mathrm{~nm} \mathrm{H}$ to $a$, where $\cos 2 a=\mu_{0}$ and $\sin 2 a=\mu_{1}$. Then, passing through $\mathrm{H}_{1}$ with rotation angle $\beta$ results in $|\Psi(\mu, \beta)\rangle^{A B}$ with desired $\mu$ and $\beta$. Using our experimental setup, the maximally entangled state can be prepared with a fidelity of 0.986 .

For preparing Werner states as in Eq. (27), we make use of an unbalanced Mach-Zehnder interferometer. In particular, two 50/ 50 beam splitters (BSs) are inserted into one branch. In the transmission path, the two-photon state is prepared as the Bell state

$$
\left|\phi^{+}\right\rangle=\frac{1}{\sqrt{2}}(|H H\rangle+|W\rangle)
$$

when the rotation angle of the $404 \mathrm{~nm} \mathrm{H}$ is set to $22.5^{\circ}$. In the reflected path, three $400 \lambda$ quartz crystals and an $\mathrm{H}$ with $22.5^{\circ}$ are used to dephase the two-photon state into a completely mixedstate $\mathbb{1}^{A B} / 4$. The ratio of the two states mixed at the output port of the second BS can be changed by the two adjustable apertures for the generation of Werner states in Eq. (27) with arbitrary $q_{w}$. Out of the state preparation module, the two photons are distributed to Alice and Bob. In fact, the two BSs are not ideally 50/50 and the transmission rate for $H$ and $V$ polarized photons are not exactly the same, resulting in a decrease of fidelity to $F=0.971$ when we prepare maximally entangled state, although we have slightly adjusted the rotation angle of the $404 \mathrm{~nm} \mathrm{H}$. It is noteworthy that in our experiments, we adopt $0 \equiv H$ and $1 \equiv V$.

In module (II) of Fig. 4d, a class of SIOs are implemented on Bob's photons, by the combination of six Hs and three BDs. For details, we refer to the Supplementary Material. In the case of b, $c$, these operations can depend on the result of measurements made on Alice's qubit. ${ }^{43,44}$

In module (III) of Fig. 4d, we perform quantum-state tomography ${ }^{45}$ to identify the target states $\left\{p_{i}, \rho_{i}\right\}$. When we conduct the experiment without assistance, the single-qubit state after the $\mathrm{IO}$ can be directly identified via the combination of two Hs, two Qs, and two PBSs in module (III). For deterministic state conversion, we directly read the total coincident counts from the two single photon detectors (SPDs); and for stochastic state conversion, we discard the counts from $K_{2}$. For experimentally determining the conversion probability in the case of stochastic conversion (SC), we also collect data in an orthogonal basis. The probability for SC can then be evaluated as

$$
P_{1}=\frac{N_{1}}{N_{\text {total }}}
$$

where $N_{1}$ denotes the total coincident counts from $K_{1}$ and $N_{\text {total }}$ denotes the total coincident counts from $K_{1}$ and $K_{2}$, in basis $\{|H\rangle,|V\rangle\}$.

When we conduct the experiments with assistance, Alice can perform arbitrary local projective measurements on her photons and broadcast the measurement outcomes to Bob. Specifically, Alice chooses the optimal measurement, which helps Bob to perform the optimal conversion. When Bob gets the information from Alice, which is either 0 or 1 , he can then implement the aforementioned IOs, obtaining the final target states.

For data collections, we used SMFs on Bob's arm and multimode fibers on Alice's arm for directing photons from space to detectors. The use of multi-mode fibers can increase and stabilize the collection effeciency of Alice's photons. On the other hand, the use of SMFs on Bob's side is preferable for cleaning up the highorder optical modes, resulting in best interference between the light beams that are displaced by the BDs. As mentioned before, the power of the $404 \mathrm{~nm}$ continuous laser is set to about $80 \mathrm{~mW}$ and the coincidence window is $4 \mathrm{~ns}$, resulting in 2000 coincident events per second. When adding white noise on Alice's arm, the coincident counts decrease to around $25 \%$ when compared with the case without noise.

\section{Experimental conversion without assistance}

For verifying the theoretical predictions of incoherent single-qubit state conversion, we experimentally initialize Bob's photon as in Eq. (25) where $r_{x}=\frac{1}{3}, r_{z}=\frac{5}{6}$ for a mixed input and $r_{x}=\frac{\sqrt{11}}{6}, r_{z}=\frac{5}{6}$ for a pure input. Our goal is now to convert the initial states using the IOs available in our experiments. In Fig. 5a, b, the experimentally obtained boundary of state space for deterministic conversion (DC) is shown for the $x-z$ plane by red cubes. The boundaries with respect to SC are shown by blue cubes. All solid lines represent theoretical predictions from Theorem 3. Also, the experimental conversion probability is depicted as a function of $x$ $z$ coordinates of the target state $\sigma$ by cylinders on the right of each plot. The solid lines represent the theoretical predictions from Eq. (6). There is a fundamental difference between pure and mixed inputs: a pure and coherent input can be converted via IOs into any qubit state at least stochastically. For mixed input, this is not the case.

Experimental conversion with assistance

To explore assisted conversions experimentally, we first let Bob share a pure entangled state $|\psi\rangle^{A B}$ with Alice, where Bob's local state is the state in Eq. (25) with Bloch coordinates $\left(0,0, \frac{5}{6}\right)$ and 
(a)

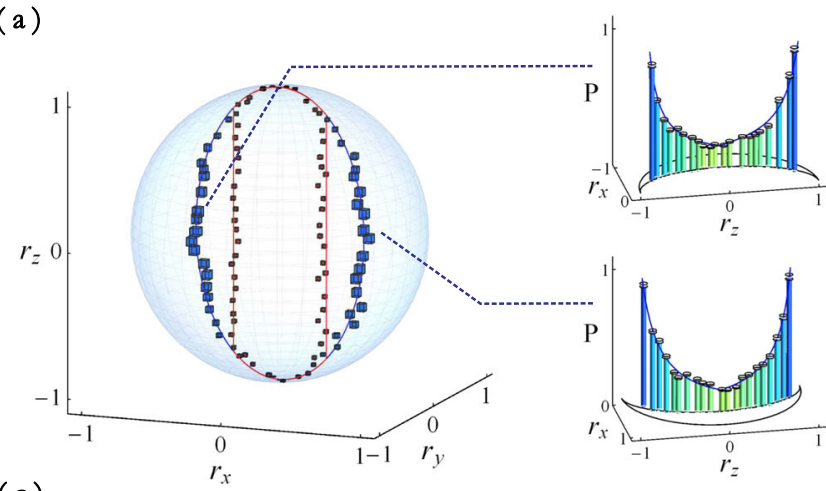

(c)

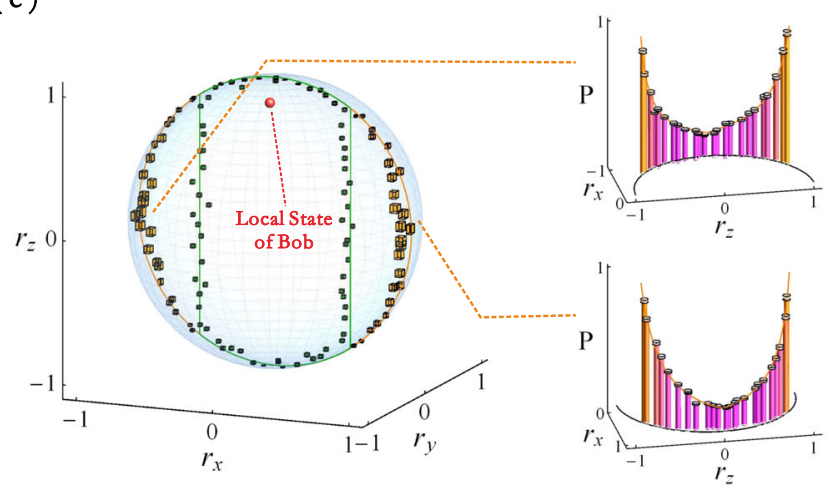

(b)

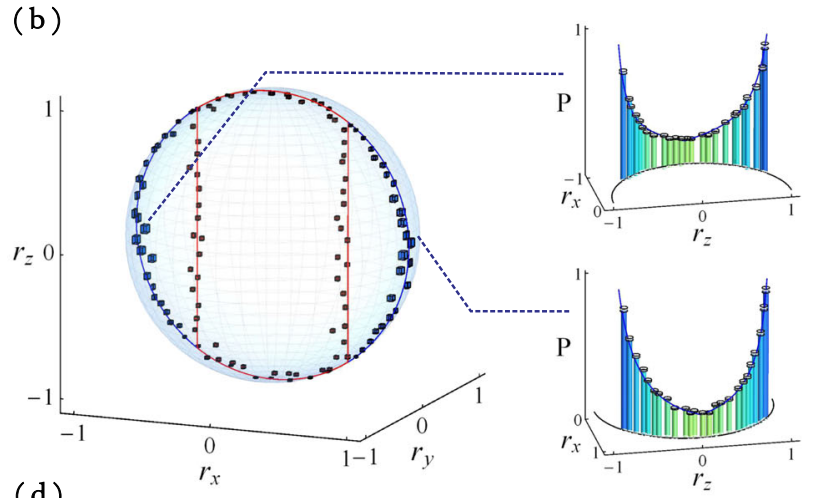

(d)

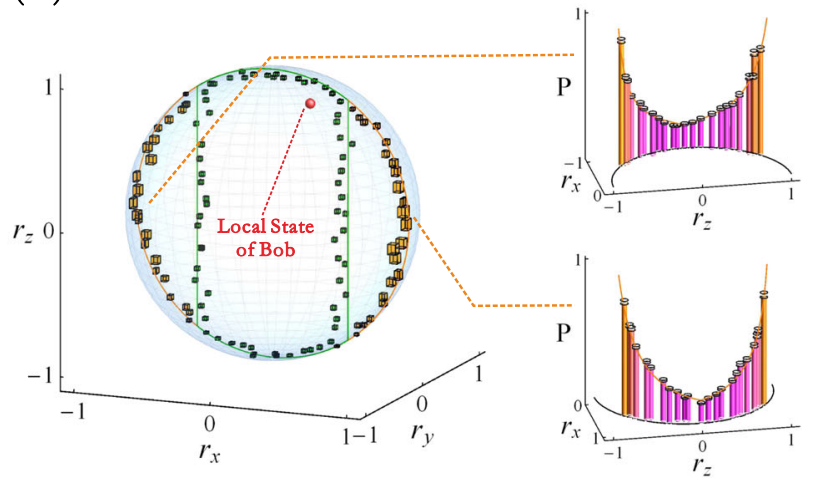

Fig. 5 Experimental results for state conversion: single-qubit states and pure two-qubit states. Experimental results for two local states for Bob as in Eq. (25) with Bloch coordinates $\left(\frac{1}{3}, 0, \frac{5}{6}\right)$ and $\left(\frac{\sqrt{11}}{6}, 0, \frac{5}{6}\right)$, are shown in $\mathbf{a}, \mathbf{b}$ without assistance from Alice. The states are prepared with high fidelity up to 0.999 . In the left of $\mathbf{a}, \mathbf{b}$, the deterministic conversion (DC) and stochastic conversion (SC) boundaries in $x-z$ plane are shown in red and blue cubes, respectively, with each side representing the variance $\delta\left\langle r_{i}\right\rangle(i=x, y, z)$ derived from Poisson distribution of single photons. In the right of $\mathbf{a}, \mathbf{b}$, conversion probabilities $P(\rho \rightarrow \sigma)$ for boundaries of $S C$ are shown with respect to the $x-z$ Bloch coordinates of the target state $\sigma^{B}$. Experimental results for two local states for Bob, with Bloch coordinates $\left(0,0, \frac{5}{6}\right)$ and $\left(\frac{1}{3}, 0, \frac{5}{6}\right)$, sharing pure entangled states $|\psi\rangle^{A B}$ with Alice, are shown in $\mathbf{c}$, $\mathbf{d}$. The experimental two-qubit states are prepared with fidelity of 0.989 and 0.982 . In the left of $\mathbf{c}, \mathbf{d}$, the DC and $\mathrm{SC}$ boundaries in $x-z$ plane are shown in green and orange cubes, respectively. In the right of $\mathbf{c}$, $\mathbf{d}$, the probabilities of conversion $P_{a}\left(|\psi\rangle^{A B} \rightarrow|\phi\rangle^{B}\right)$ are shown with respect to the $x-z$ Bloch coordinates of the target state $|\phi\rangle^{B}$. Solid lines represent theoretical predictions.

$\left(\frac{1}{3}, 0, \frac{5}{6}\right)$. Our experimental results are shown in Fig. $5 c$, d. Remarkably, due to Theorem 6 , the probability for assisted conversion only depends on the $z$-coordinate of the initial state, which explains the close similarity of both the state spaces and conversion probabilities in Fig. 5c, d. We also experimentally test two Werner states, one with entanglement and one without. In Fig. 6, the DC boundaries are shown in purple and blue for the two states. In accordance with Eq. (13), resorting to SCs does not allow to prepare additional states.

\section{Comparison of the experimental protocols}

Compared with DC, Bob can obtain more states when he allows for SC and even obtain all qubit states by taking advantage of assistance. This can be seen in Fig. 7, where we compare our results for the different protocols. In Fig. 7a, we experimentally show the boundary of accessible state space, both deterministically and stochastically, with and without assistance. Noting that the $\ell_{1}$ norm of coherence ${ }^{10} C_{\ell_{1}}$ reads

$C_{\ell_{1}}(\rho)=\sum_{i \neq j}\left|\rho_{i j}\right|=r$

for qubit states, we can obtain a relation between the coherence of a target state and the probability to obtain it using IOs. The experimental results are shown in Fig. 7b. Although local coherence can never be increased deterministically, we can still exceed the original coherence at the expense of success probability. A maximally coherent state $|+\rangle$ can be obtained by utilizing $\mathrm{SC}$ and assistance.

\section{DISCUSSION}

In this work we study the problem of quantum-state conversion within the resource theory of quantum coherence, both theoretically and experimentally. The state-conversion problem is important in any resource theory, as it determines the value of states for protocols using the resource under study. The result presented here are a significant generalization of recent results on single-shot coherence theory ${ }^{16,17,20-22}$ and single-shot resource theories in general, ${ }^{23}$ and include necessary conditions on the existence of SCs, which we generalized to higher dimensions.

In most resource theories, one is also interested in the possibilities of asymptotic state conversion, where many instances of the initial and final state are available. As we have shown, our results also pave the way towards a complete solution of this problem: our single-shot conversion rate gives a lower bound on the asymptotic conversion rate, which is in some areas significantly better than the best previously known bound. ${ }^{11}$ In addition, it coincides for some states with an upper bound from ref. ${ }^{11}$ solving the asymptotic conversion problem in these cases. Moreover, the results allow us to investigate the irreversibility of coherence theory in the asymptotic limit and to determine the possible distillable coherence for fixed coherence cost. 


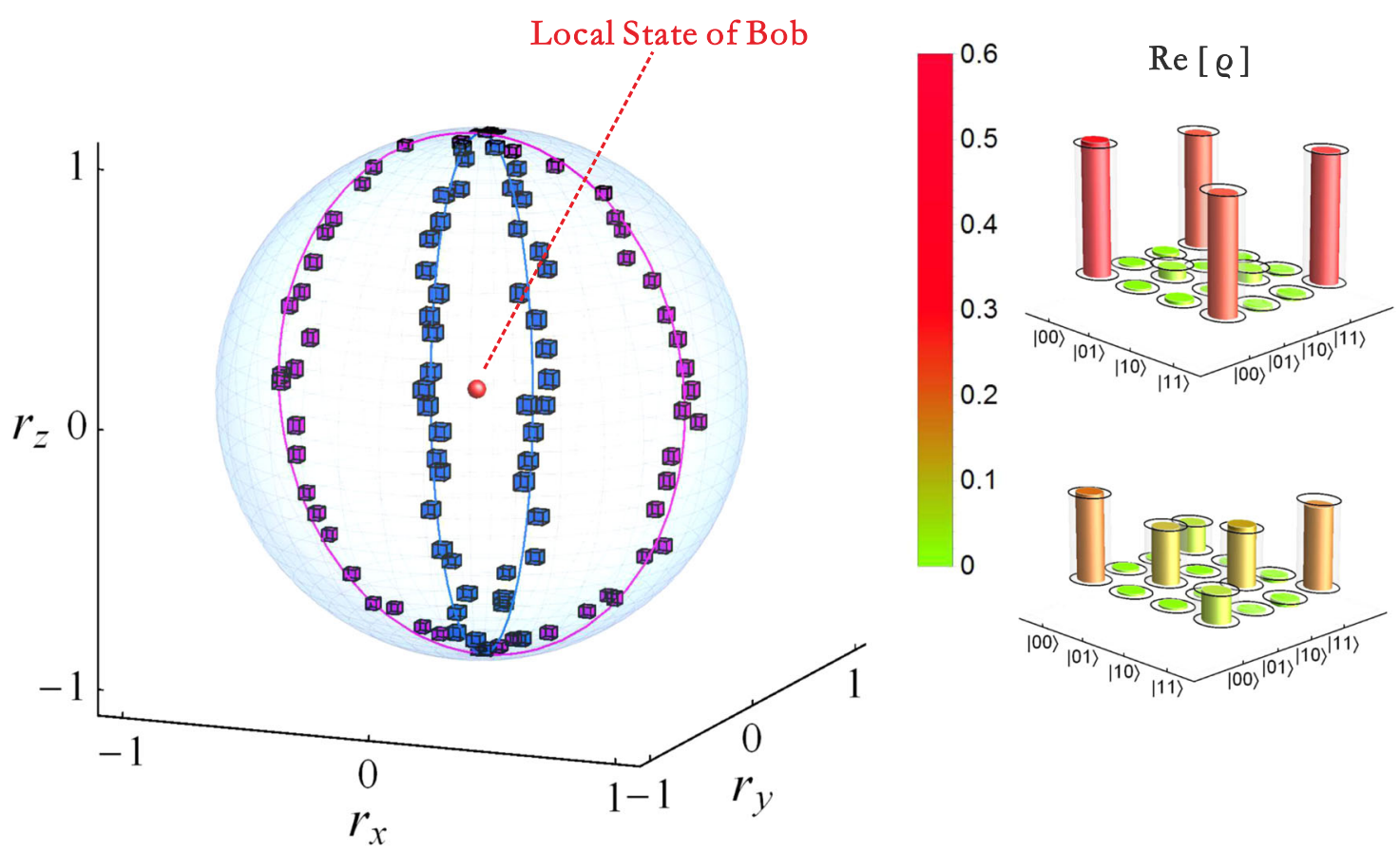

Fig. 6 Experimental results for assisted incoherent state conversion: noisy two-qubit states. Experimental results for local states for Bob, $\rho^{B}=\frac{1}{2} \mathbb{1}$, sharing pure entangled states $|\psi\rangle^{A B}$ with Alice, subjected to a controllable proportion of white noise. Although Bob will find his system in a maximally mixed state with zero coherence, he can prepare certain coherent states if he takes advantage of Alice's assistance. In our experiment, we use two Werner states with $q_{\mathrm{w}}=0.8245$ and 0.2075 . In the right, the real parts of the tomographically reconstructed quantum states $\rho_{\mathrm{w}}^{A B}$ are shown, with a fidelity of 0.986 and 0.997 , respectively. In the left, the DC boundaries in $x-z$ plane for conversions $\rho_{\mathrm{w}}^{A B} \rightarrow \sigma^{B}$ are shown in purple and blue, respectively.

(a)

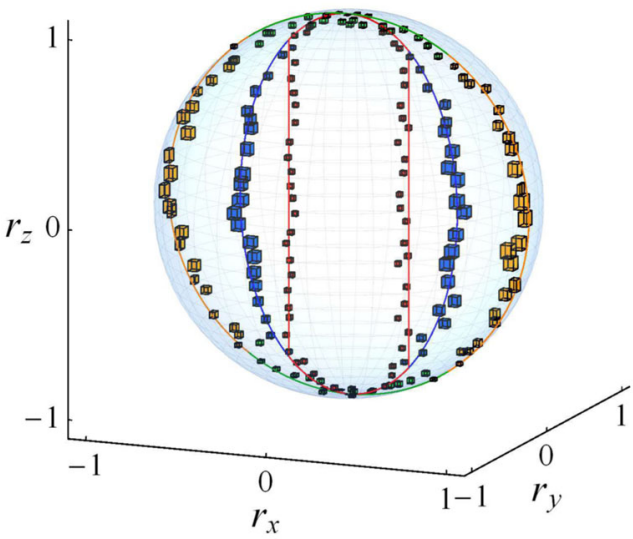

(b ) $P_{\max }$

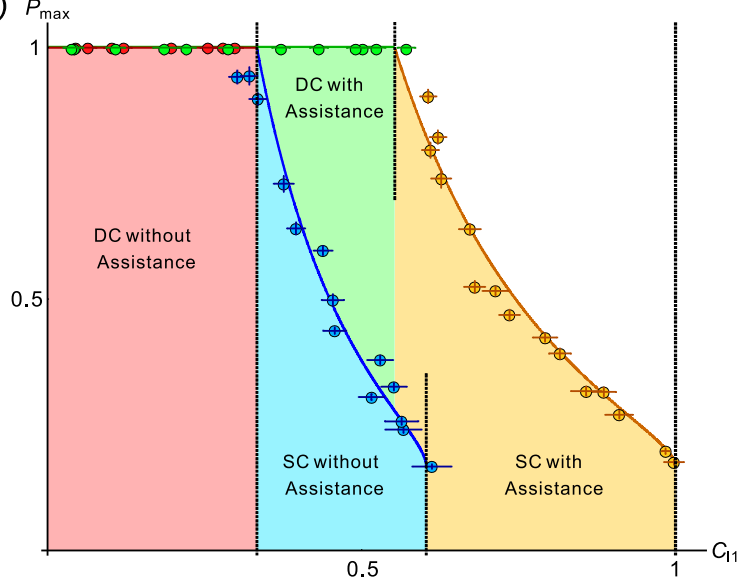

Fig. 7 Experimental results for showing the capability of enlarging conversion boundaries via different protocols. The local state of Bob is experimentally prepared as $\rho^{B}=\frac{1}{2}\left(1+\frac{1}{3} \sigma_{x}+\frac{5}{6} \sigma_{z}\right)$. In a, we can see that the accessible states of Bob can be enlarged by using different conversion protocols; the red boundary can be achieved via DC without assistance, which shows the basic capability of local incoherent conversion. When we use SC, without assistance, we can make the conversion boundary larger, shown as blue. Combining the boundary of SC and DC, we obtain an ellipsoid in the Bloch space. With assistance from a pure source, we can enlarge the conversion boundary to the surface of the Bloch sphere. The boundaries of assisted conversion, both DC and SC, are shown as green and orange, respectively. As the boundaries are rotationally invariant with respect to $z$, we focus on the $x-z$ plane by taking a round cross-section. In $\mathbf{b}$, the maximal success probabilities vs. obtained $\ell_{1}$ norm of coherence are plotted for these different protocols.

Experimentally implementing non-unitary IOs, we demonstrated that a quantum optical experiment can closely achieve the expected optimal conversion rates. The corresponding optical setup should be seen as a building block for more general transformations, also going beyond single qubits and IOs. The results presented in this work can then serve as benchmarks for these more advanced setups.

\section{DATA AVAILABILITY}

All data not included in the paper and its Supplementary Material are available upon reasonable request from the corresponding authors.

Received: 1 October 2019; Accepted: 2 January 2020;

Published online: 13 February 2020 


\section{REFERENCES}

1. Horodecki, R., Horodecki, P., Horodecki, M. \& Horodecki, K. Quantum entanglement. Rev. Mod. Phys. 81, 865-942 (2009).

2. Bennett, C. H. et al. Teleporting an unknown quantum state via dual classical and einstein-podolsky-rosen channels. Phys. Rev. Lett. 70, 1895-1899 (1993).

3. Einstein, A., Podolsky, B. \& Rosen, N. Can quantum-mechanical description of physical reality be considered complete? Phys. Rev. 47, 777-780 (1935).

4. Vedral, V., Plenio, M. B., Rippin, M. A. \& Knight, P. L. Quantifying entanglement. Phys. Rev. Lett. 78, 2275-2279 (1997).

5. Plenio, M. B. \& Virmani, S. An introduction to entanglement measures. Quant. Inf. Comp. 7, 1-51 (2007).

6. Zurek, W. H. Decoherence, einselection, and the quantum origins of the classical. Rev. Mod. Phys. 75, 715-775 (2003).

7. Bromley, T. R., Cianciaruso, M. \& Adesso, G. Frozen quantum coherence. Phys. Rev. Lett. 114, 210401 (2015).

8. Aberg, J. Quantifying superposition. Preprint at http://arxiv.org/abs/quant-ph/ 0612146 (2006)

9. Levi, F. \& Mintert, F. A quantitative theory of coherent delocalization. New J. Phys. 16, 033007 (2014).

10. Baumgratz, T., Cramer, M. \& Plenio, M. B. Quantifying coherence. Phys. Rev. Lett. 113, 140401 (2014)

11. Winter, A. \& Yang, D. Operational resource theory of coherence. Phys. Rev. Lett 116, 120404 (2016).

12. Chitambar, E. \& Gour, G. Critical examination of incoherent operations and a physically consistent resource theory of quantum coherence. Phys. Rev. Lett. 117, 030401 (2016).

13. Yadin, B., Ma, J., Girolami, D., Gu, M. \& Vedral, V. Quantum processes which do not use coherence. Phys. Rev. X 6, 041028 (2016).

14. Streltsov, A., Adesso, G. \& Plenio, M. B. Colloquium: quantum coherence as a resource. Rev. Mod. Phys. 89, 041003 (2017).

15. Zhu, H., Ma, Z., Cao, Z., Fei, S.-M. \& Vedral, V. Operational one-to-one mapping between coherence and entanglement measures. Phys. Rev. A 96, 032316 (2017).

16. Streltsov, A., Rana, S., Boes, P. \& Eisert, J. Structure of the resource theory of quantum coherence. Phys. Rev. Lett. 119, 140402 (2017).

17. Shi, H.-L. et al. Coherence transformations in single qubit systems. Sci. Rep. 7, 14806 (2017)

18. Du, S., Bai, Z. \& Qi, X. Coherence measures and optimal conversion for coherent states. Quant. Inf. Comp. 15, 1307-1316 (2015).

19. Du, S., Bai, Z. \& Qi, X. Erratum: To "coherence measures and optimal conversion for coherent states" [Quant. Inf. Comp., vol. 15(2015), 1307-1316]. Quant. Inf. Comp. 17, 503-505 (2017).

20. Regula, B., Fang, K., Wang, X. \& Adesso, G. One-shot coherence distillation. Phys. Rev. Lett. 121, 010401 (2018).

21. Vijayan, M. K., Chitambar, E. \& Hsieh, M.-H. One-shot assisted concentration of coherence. J. Phys. A 51, 414001 (2018).

22. Fang, K., Wang, X., Lami, L., Regula, B. \& Adesso, G. Probabilistic distillation of quantum coherence. Phys. Rev. Lett. 121, 070404 (2018).

23. Gour, G. Quantum resource theories in the single-shot regime. Phys. Rev. A 95 062314 (2017).

24. Chitambar, E. \& Gour, G. Quantum resource theories. Rev. Mod. Phys. 91, 025001 (2019).

25. Napoli, C. et al. Robustness of coherence: an operational and observable measure of quantum coherence. Phys. Rev. Lett. 116, 150502 (2016).

26. Marvian, I. \& Spekkens, R. W. How to quantify coherence: distinguishing speakable and unspeakable notions. Phys. Rev. A 94, 052324 (2016).

27. Hillery, M. Coherence as a resource in decision problems: the Deutsch-Jozsa algorithm and a variation. Phys. Rev. A 93, 012111 (2016).

28. Matera, J. M., Egloff, D., Killoran, N. \& Plenio, M. B. Coherent control of quantum systems as a resource theory. Quant. Sci. Technol. 1, 01 LT01 (2016).

29. Huelga, S. F. \& Plenio, M. B. Vibrations, quanta and biology. Contemp. Phys. 54, 181-207 (2013).

30. Theurer, T., Killoran, N., Egloff, D. \& Plenio, M. B. Resource theory of superposition. Phys. Rev. Lett. 119, 230401 (2017).

31. Chitambar, E. \& Gour, G. Comparison of incoherent operations and measures of coherence. Phys. Rev. A 94, 052336 (2016).

32. Piani, M. et al. Robustness of asymmetry and coherence of quantum states. Phys. Rev. A 93, 042107 (2016)

33. Killoran, N., Steinhoff, F. E. S. \& Plenio, M. B. Converting nonclassicality into entanglement. Phys. Rev. Lett. 116, 080402 (2016).

34. Chin, S. Coherence number as a discrete quantum resource. Phys. Rev. A 96, 042336 (2017).

35. Chin, S. Generalized coherence concurrence and path distinguishability. J. Phys. A 50, 475302 (2017).
36. Chitambar, E. et al. Assisted distillation of quantum coherence. Phys. Rev. Lett. 116, 070402 (2016).

37. Streltsov, A., Rana, S., Bera, M. N. \& Lewenstein, M. Towards resource theory of coherence in distributed scenarios. Phys. Rev. X 7, 011024 (2017).

38. Yuan, X., Zhou, H., Cao, Z. \& Ma, X. Intrinsic randomness as a measure of quantum coherence. Phys. Rev. A 92, 022124 (2015).

39. Horodecki, M., Horodecki, P. \& Horodecki, R. Mixed-state entanglement and distillation: Is there a "bound" entanglement in nature? Phys. Rev. Lett. 80 5239-5242 (1998).

40. Horodecki, M., Oppenheim, J. \& Horodecki, R. Are the laws of entanglement theory thermodynamical? Phys. Rev. Lett. 89, 240403 (2002).

41. Brandão, F. G. S. L. \& Gour, G. Reversible framework for quantum resource theories. Phys. Rev. Lett. 115, 070503 (2015).

42. Streltsov, A., Singh, U., Dhar, H. S., Bera, M. N. \& Adesso, G. Measuring quantum coherence with entanglement. Phys. Rev. Lett. 115, 020403 (2015).

43. Wu, K.-D. et al. Experimentally obtaining maximal coherence via assisted distillation process. Optica 4, 454-459 (2017).

44. Wu, K.-D. et al. Experimental cyclic interconversion between coherence and quantum correlations. Phys. Rev. Lett. 121, 050401 (2018).

45. Qi, B. et al. Quantum state tomography via linear regression estimation. Sci. Rep. 3, 3496 (2013).

\section{ACKNOWLEDGEMENTS}

We acknowledge useful discussions with Dario Egloff, Swapan Rana, Christine Silberhorn, and Jan Sperling. K.-D.W., G.-Y.X., C.-F.L., and G.-C.G. acknowledge support from the National Nature Science Foundation of China (NSFC; 11574291 and 11774334), National Key R\&D Program (2016YFA0301700), and Anhui Initiative in Quantum Information Technologies. M.B.P. and T.T. acknowledge support by the ERC Synergy Grant BioQ (grant number 319130) and the BMBF project Q.Link.X. A.S. and K.-D.W. acknowledge financial support by the "Quantum Optical Technologies" project, carried out within the International Research Agendas program of the Foundation for Polish Science co-financed by the European Union under the European Regional Development Fund.

\section{AUTHOR CONTRIBUTIONS}

All authors contributed significantly to this work.

\section{COMPETING INTERESTS}

The authors declare no competing interests.

\section{ADDITIONAL INFORMATION}

Supplementary information is available for this paper at https://doi.org/10.1038/ s41534-020-0250-z.

Correspondence and requests for materials should be addressed to G.-Y.X. or A.S.

Reprints and permission information is available at http://www.nature.com/ reprints

Publisher's note Springer Nature remains neutral with regard to jurisdictional claims in published maps and institutional affiliations.

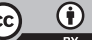

Open Access This article is licensed under a Creative Commons Attribution 4.0 International License, which permits use, sharing, adaptation, distribution and reproduction in any medium or format, as long as you give appropriate credit to the original author(s) and the source, provide a link to the Creative Commons license, and indicate if changes were made. The images or other third party material in this article are included in the article's Creative Commons license, unless indicated otherwise in a credit line to the material. If material is not included in the article's Creative Commons license and your intended use is not permitted by statutory regulation or exceeds the permitted use, you will need to obtain permission directly from the copyright holder. To view a copy of this license, visit http://creativecommons. org/licenses/by/4.0/.

(c) The Author(s) 2020 